\title{
Effects of micro-algae commonly used in aquaculture on acyl-homoserine lactone quorum sensing.
}

\begin{abstract}
Quorum sensing, bacterial cell-to-cell communication with small signal molecules such as acyl-homoserine lactones, regulates the virulence of many pathogenic bacteria. Therefore, interfering with quorum sensing is currently being explored as a novel biocontrol strategy to fight bacterial infections. In this study, the effects of 19 micro-algal strains on acylhomoserine lactone-regulated phenotypes of three reporter strains were investigated. Two freshwater micro-algae inhibited violacein production of quorum sensing reporter strain Chromobacterium violaceum CV026. Further tests using Escherichia coli JB523 showed that micro-algal extracts inhibited or stimulated quorum sensing, depending on the algal strain. One freshwater and five marine algae showed quorum sensing inhibitory activity, whereas two algae stimulated quorum sensing-regulated gene expression. Micro-algal strains that showed inhibitory activity in the previous assays also inhibited acyl-homoserine lactoneregulated bioluminescence in the aquaculture pathogen Vibrio harveyi. The growth of all reporter strains was found to be unaffected by the micro-algal samples. The most promising micro-algal strain was found to be Chlorella saccharophila CCAP211/48, as its extracts inhibited quorum sensing-regulated gene expression in all three reporter strains.
\end{abstract}

Keyword: Quorum sensing; Microalgae; Quorum sensing interference; Luminescent vibrios. 